\title{
Restablecimiento de una nueva normalidad biomecánica en las graves deformidades de la rodilla
}

\author{
DENARO V., CANCILLERI F., MARINOZZI A., \\ STELLATO GFM., TAGLIERI E. \\ Libera Universitá "Campus Bio-Medico" \\ Area di Ortopedia y Traumatologia \\ Roma- Italia
}

\begin{abstract}
Resumen
En las graves alteraciones morfo-estructurales de la rodilla, con inestabilidad en varusvalgus, las prótesis vinculadas a bisagra simple, tendían a fallar. El presente trabajo se propone evidenciar que, en tales situaciones, la prótesis vinculada a rotación representa la solución más idónea para el restablecimiento de una nueva normalidad. 18 pacientes (13 mujeres y 5 hombres) con edad media de 68 años han sido sometidos a intervención para colocar prótesis de rodilla. Ha sido utilizado el implante Endo-Model® de Waldemar Link®, prótesis vinculada a rotación. El follow-up medio es de 19 meses con controles a 2, 3, 6 y 12 meses y después anuales. Los resultados han sido: óptimo para 17 pacientes y bueno para 1 paciente, en el cual se ha verificado la ruptura post-operatoria del tendón cuadricipital. Nuestra casuística muestra resultados comparables a los de otros autores. No hemos tenido movilizaciones asépticas o sépticas, ni complicaciones médicas intraoperatorias y post-operatorias. Consideramos que, en presencia de la correcta indicación clínica, la utilización de la prótesis vinculada a rotación es preferible a la de deslizamiento porque disminuye el riesgo de una movilización precoz del implante y del desgaste anormal de los componentes protésicos, restableciendo una nueva normalidad biomecánica.
\end{abstract}

Palabras clave: rodilla, prótesis de rodilla; prótesis vinculada a rotación.

\section{Introducción}

En la cadena cinética del miembro inferior la rodilla, en condiciones fisiológicas, explica su función gracias a la morfología articular y a un delicado equilibrio cápsulo-ligamentoso sobre el cual funcionan los componentes musculares. Como se observa, la articulación posee un amplio grado de libertad, la flexo-extensión, a la cual

E-mail: f.cancilleri@unicampus.it accesoriamente se agregan la rotación externa e interna y la translación de la tibia que se cumplen solamente durante la flexión. La combinación de estos movimientos hace posibles dos condiciones aparentemente contradictorias: la posesión de una gran estabilidad en extensión completa y una gran movilidad, a partir de un cierto grado de flexión, necesaria para garantizar el arco deambulatorio desde el simple camino a la carrera. En la práctica 
clínica, se verifican condiciones de graves alteraciones morfo-estructurales de la rodilla, de origen degenerativo $\mathrm{o}$ post-traumático que comprometen seriamente la capacidad deambulatoria y ponen serios problemas para obtener una nueva normalidad protésica. Se intuye, por lo tanto, cómo en la evolución de la cirugía protésica se tienda al restablecimiento, lo más fiel posible, de la fisiología articular en el mayor respeto de las estructuras músculo-ligamentosas. En estas condiciones la dificultad mayor deriva del restablecimiento de una correcta tensión ligamentosa, esencial a los fines de la estabilidad articular y, por consiguiente, es indicado el recurso a una prótesis vinculada ${ }^{1,2}$.

En la mayor parte de las primeras prótesis vinculadas ( Shiers ${ }^{3,4}$, Walldius ${ }^{5}$, Young ${ }^{6,7}$, Guepar ${ }^{8,9}$ y Lagrange-Letournelle) se realizaba con preponderancia una bisagra simple para obtener una estabilidad intrínseca primaria, sacrificando así el componente de rotación de la tibia respecto al fémur. Estos defectos biomecánicos están en la base de los escasos resultados obtenidos, además de los inconvenientes de una no correcta orientación anatómica de las raíces de la prótesis dirigido a la transmisión de las fuerzas de carga y a la necesidad de efectuar macizas resecciones óseas. Para evitar tales límites desde 1969 al menos dos grupos de cirujanos alemanes (Nieder y Blauth) $)^{10,} 11$ han iniciado el proyecto de un tipo de prótesis vinculado a rotación, en el cual sea respetada la orientación anatómica de las raíces y el componente rotatorio de la tibia.

\section{Materiales y Métodos}

Cuando la articulación de la rodilla presenta una deformidad en varus o en valgus igual o mayor de $20^{\circ}$, o cuando los ligamentos colaterales muestran una marcada laxitud, la deambulación resulta gravemente alterada $o$, en algunos casos, directamente imposible sin la ayuda de tutor (Figura 1). En estas condiciones el implante de una prótesis de deslizamiento resultaría inadecuado por la dificultad de restablecer la correcta tensión de los colaterales, fundamentales para conseguir la estabilidad de la prótesis y elemento determinante, en caso de déficit, para el éxito de la intervención. Son éstas las situaciones clínicas en las cuales reconocemos la indicación para el empleo de una prótesis vinculada a rotación.

Para nuestros implantes hemos escogido el tipo
Endo-Model de Waldemar Link, que representa la evolución del proyecto del grupo de Endo Klinik a partir del primer modelo que fue denominado St. Georg ${ }^{10}$.

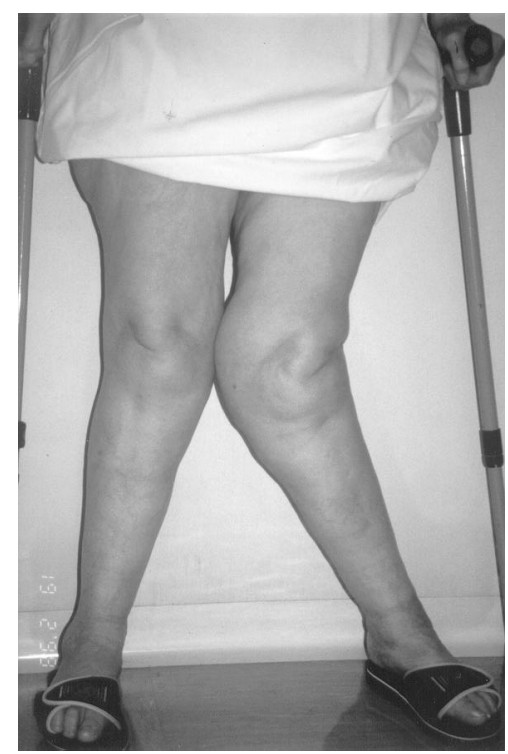

Figura 1. Grave deformidad en valgus con deambulación gravemente alterada

La terminología de prótesis "vinculada a rotación" expresa bien sus dos características fundamentales: la estabilidad intrínseca elevada, unida a una gran articularidad. Esta última no sólo en flexión sino que posee también un movimiento de rotación que aumenta con el progreso de la flexión misma. Esto se obtiene gracias a un particular sistema de acoplamiento, mediante una juntura articulada, entre el componente femoral y el tibial (Figura 2). Estas características conjugan las ventajas de una prótesis de bisagra (elevada estabilidad) con las de una prótesis de deslizamiento (elevada articularidad). Al mismo tiempo limitan las desventajas de la bisagra pura reduciendo drásticamente el stress torsional de los componentes de la prótesis debido a la ausencia de rotación con consiguiente riesgo de movilización, y los de la inestabilidad provocada por un deficitario aparato capsulo-ligamentoso, causa del fallo de una prótesis a deslizamiento ${ }^{10}$. Se subraya que la raíz femoral, para permitir un restablecimiento del eje fisiológico de carga de la rodilla, posee un alineamiento en desviación de $6^{\circ}$ respecto al componente tibial. El implante prevé la cementación de ambos componentes y no prevé la sustitución de la rótula. 


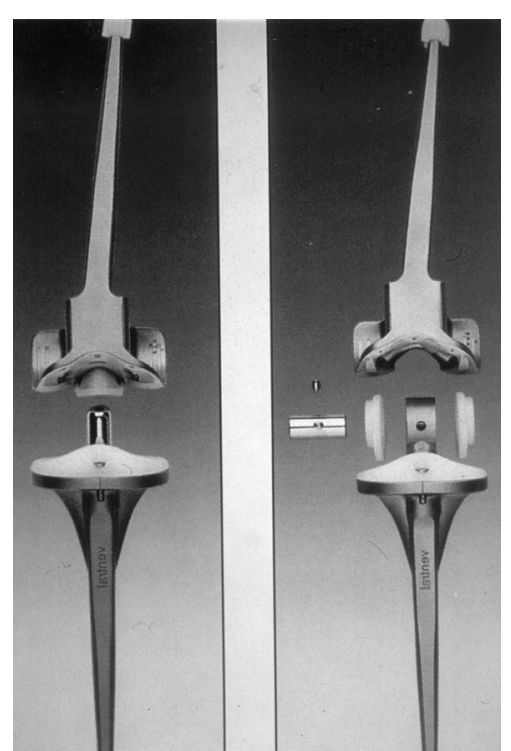

Figura 2. Imagen de la prótesis vinculada a rotación

Hemos implantado esta prótesis en 18 casos de grave deformidad de la rodilla con grave pérdida de la capacidad deambulatoria: 13 mujeres y 5 hombres con edad media de 68 años. En tres casos ha sido efectuado un implante bilateral. El followup medio es de 19 meses, con un máximo de 38 y un mínimo de 4.

\section{Resultados}

Los resultados han sido valorados según los siguientes parámetros:

1. Restablecimiento del eje fisiológico

2. Articularidad $\left(0^{\circ}-100^{\circ}\right)$

3. Ausencia de dolor.

4. Recuperación de la deambulación libre

5. Capacidad de subir y bajar las escaleras

6. Capacidad de levantarse de una silla sin ayuda de los brazos

7. Satisfacción por parte del Paciente

Se ha seguido un riguroso calendario de los controles post-operatorios a 1, 3, 6 y 12 meses y, sucesivamente, cada año. El control prevé la ejecución de un radiograma en ortostatismo en proyección standard.

En 1 caso hemos tenido una complicación, en el inmediato post-operatorio, por la ruptura del tendón cuadricipital. En los restantes casos la rehabilitación pasiva mediante CPM de rodilla se ha iniciado el $2^{\circ}$ día y han deambulado, sin limitación de carga, entre el $3^{\circ}$ y el $4^{\circ}$ día. En el $8^{\circ}$ día han alcanzado los $90^{\circ}$ de flexión real.
El paciente en el cual se produjo la ruptura del tendón cuadricipital consiguió los parámetros 2, 4 , 5 y 6 después de 3,5 meses de la intervención. Por lo tanto se han obtenido 17 resultados óptimos (adquisición de todos los parámetros en el plazo de un mes) y 1 bueno.

En la fotos 3, 4, 5, 6, y 7 se presenta un caso representativo.

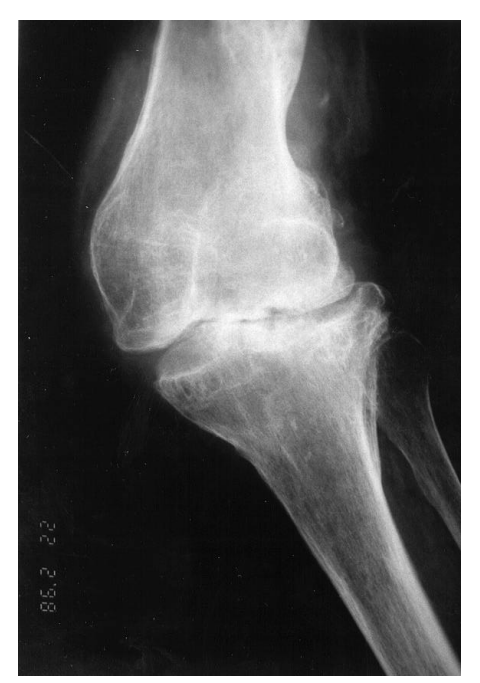

Figura 3. Imagen radiológica correspondiente al caso de la Figura 1.

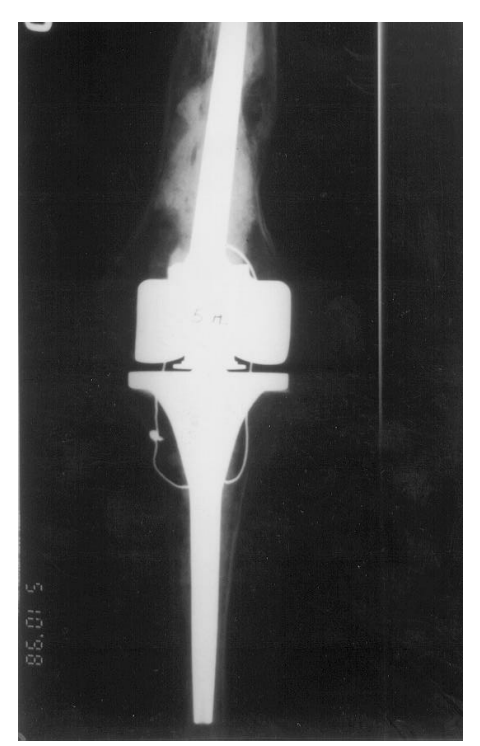

Figura 4. Control post-operatorio después de 5 meses donde se demuestra la buena posición de la prótesis. El cerclaje y la cambra se han colocado para reparar la ruptura del tendón cuadricipital 


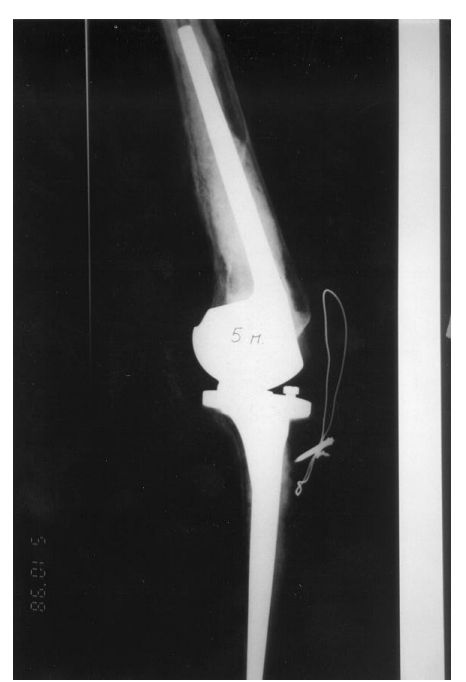

Figura 5. Control post-operatorio después de 5 meses donde se demuestra la buena posición de la prótesis. El cerclaje y la cambra se han colocado para reparar la ruptura del tendón cuadricipital

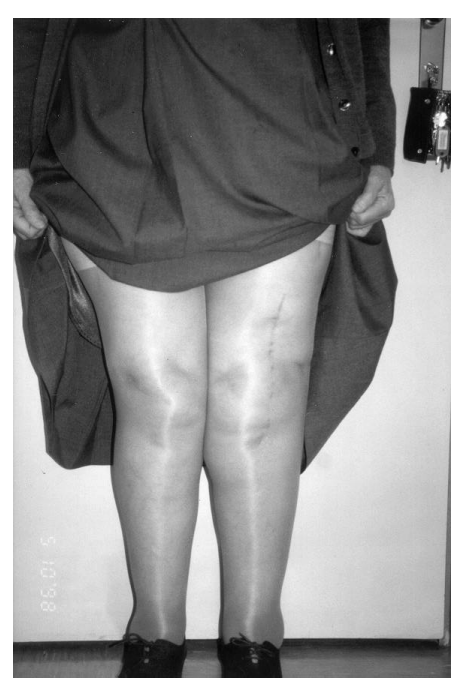

Figura 6. Restablecimiento del eje fisiológico

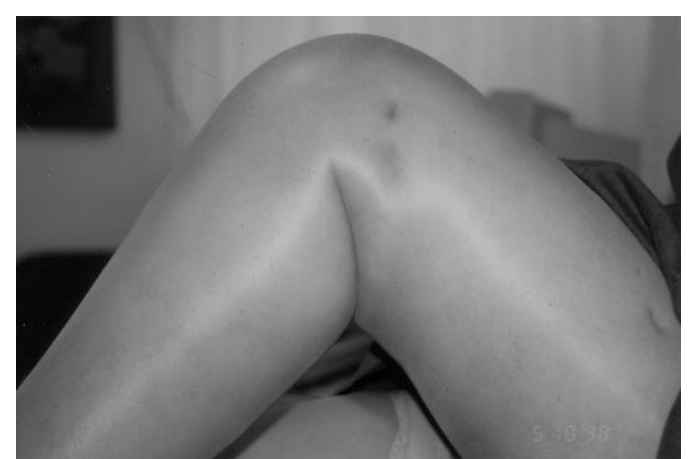

Figura 7. Recuperación de la articulación

\section{Discusión}

Los resultados obtenidos son comparables, aunque con una casuística necesariamente limitada, a los de otros Autores con casuística mucho más amplia $^{12,13,14}$.

En los controles hasta ahora seguidos ninguna prótesis presenta signos de movilización aséptica.

En el período post-operatorio inmediato y en el tardío no han aparecido complicaciones médicas. Bohm, en el trabajo ya citado, con una casuística mucho más amplia, reporta dos fallecimientos por embolia pulmonar, cinco casos de trombosis venosa profunda, un caso de pulmonía y de colestasis en un paciente con hepatitis $\mathrm{B}$ crónica $\mathrm{y}$, finalmente, un caso de ileo paralítico.

No hemos tenido la complicación de la fractura intra-operatoria de los cóndilos femorales en el momento de la preparación del alojamiento del componente protésico femoral ${ }^{15}$.

Respecto a las resecciones óseas necesarias para el montaje de la prótesis hay que mencionar que éstas no impiden que, en caso de fallo aséptico, se pueda proceder a la colocación de un segundo implante sin particulares problemas.

Finalmente, en ningún caso se ha verificado infección.

\section{Conclusiones}

Consideramos que el empleo de la prótesis vinculada a rotación se debe limitar a ciertas condiciones clínicas, como las graves deformidades de alineamiento axial de la rodilla y en algunas situaciones de particular laxitud ligamentosa no obstante sin marcada desviación axial, en las cuales una prótesis de deslizamiento requeriría una intervención quirúrgica más cuidadosa y de mayor duración, con el riesgo de que los dos componentes puedan quedar laxos o excesivamente ajustados. En el primer caso se tendría un elevado riesgo de movilización precoz del implante o de un desgaste anormal del Polietileno; en el segundo no se obtendría un válido restablecimiento de una nueva normalidad biomecánica. Por lo tanto, suponiendo la correcta indicación clínica, el recurso a la prótesis vinculada a rotación representa una válida elección para conciliar el correcto restablecimiento del eje articular con la exigencia de garantizar al paciente una recuperación rápida de la autonomía deambulatoria asociada a una estabilidad primaria inmediata. 


\section{Bibliografía}

1. Gallannaugh $\mathbf{C}$ : The Attenborough and Gallannaugh knee prostheses for total knee arthroplasty: a comparison and survival analysis. Clin Orthop 1992;281:177-88

2. Bohm P, Holy T: Is there a future for hinged prostheses in primary total knee arthroplasty? J Bone Joint Surg (Br) 1998;80-B:302-9

3. Shiers LGP: Arthroplasty of the knee: preliminary report of a new method. J Bone Joint Surg (Br) 1954;36B:553-60

4. Watson JR, Hill RCJ: The Shiers arthroplasty of the knee. . J Bone Joint Surg (Br) 1976;58-B:300-4

5. Walldius B: Arthroplasty of the knee using and endoprosthesis: 8 years experience. Acta Orthop Scand 1960;30:137-48

6. Young HH: Use of a hinged Vitallium prosthesis for arthroplasty of the knee; a preliminary report. $J$ Bone Joint Surg (Am) 1963;45-A:1627-42

7. Young HH: Use of a hinged Vitallium prosthesis (Young type) for arthroplasty of the knee. . J Bone Joint Surg (Am) 1971;53-A:1658-9

8. Deburge A: Guepar hinge prosthesis: complications and results with two years followup. Clin Orthop 1976;120:47-53

9. Jones EC, Insall JN, Inglis AE, Ranawat CS:
GUEPAR knee arthroplasty results and late complications. Clin Orthop 1979;140:145-52

10. Nieder E: Sled prosthesis, rotating knee and hinge prosthesis model St. Georg $\AA$ and Endo-Model $\AA$, Orthopade 1991;20:170-80

11. Blauth W: Uber eine neue KniegelenkTotalprothese. Med Orthop Technik 1974;94:65-7

12. Niwa S, Yoshino S, Kurosaka M, Shino K, Yamamoto S: Reconstruction of the Knee Joint. Ten to Twenty Years of Arthroplasty at the Endo-Klinik: A Report on the long-term Follow-up of the St. Georg Hinge and the Medium-term Follow-up of the Rotating Knee Endo-Model. (By Engelbrecht E, Nieder E, Kluber D; from page 186 to 199). Springer-Verlag, 1979

13. Heer TE: Results of Total Knee Revision using the LINK Endo-Model Rotating Prosthesis. Joint Implant Surgeons 1993; vol. 20

14. Nieder E: Rotating Hinge Prosthesis Endo-Model: Mid-term results of the 1837 cases at primary knee arthroplasty. Conference to be held at the $39^{\text {th }}$ Annual Spring Meeting of the Korean Orthopaedic Association. Kjungju, Korea, 13-15 April, 1995

15. Ghisellini F, Ceffa R: Le protesi Endomodell nei reimpianti settici e asettici e nei casi particolari. Esperienza personale. Il Ginocchio 1997; vol. 17:6973 